\title{
Dynamics of structural and functional changes in gut microbiota during treatment with a microalgal $\beta$ - glucan, paramylon and the impact on gut inflammation
}

Harrison Taylor

Medical University of South Carolina

Radhika Gudi

Medical University of South Carolina

Robert Brown

Medical University of South Carolina

Chenthamarakshan Vasu ( $\nabla$ vasu@musc.edu)

Medical University of South Carolina https://orcid.org/0000-0002-1772-6508

\section{Research}

Keywords: Microbiota, Paramylon, $\beta$-glucan, gut mucosa, gut inflammation, immune modulation, immune regulation

Posted Date: May 23rd, 2020

DOI: https://doi.org/10.21203/rs.3.rs-29113/v1

License: (1) This work is licensed under a Creative Commons Attribution 4.0 International License.

Read Full License 


\section{Abstract \\ Background}

Previously, we have shown that oral administration of yeast derived $\beta-1,3 / 1,6-D$-glucan, a complex dietary polysaccharide, enhances immune regulation, reduces gut inflammation and alters the composition of the gut microbiota. However, it is not known if other structurally distinct $\beta$-glucans have similar properties. The aim of this study was, using B6 mice, to investigate the ability of a microalgae derived $\beta-1,3-D-$ glucan, paramylon (PM), a widely used dietary supplement, in shaping the gut microbiota and modulating the susceptibility to gut inflammation.

\section{Results}

The community structure within the gut microbiota, based on 16S rRNA sequencing data, showed progressive changes including selective enrichment of specific communities and lowered community richness and diversity during prolonged oral treatment with PM. Compared to control mice, the gut microbiota of PM treated mice had significantly higher abundance of Verrucomicrobia $(+16.46 \%$; $p=$ $0.002)$ and lower abundance of Firmicutes $(-7.53 \% ; p=0.0147)$. Specific taxa that were significantly more abundant in PM treated mice include Akkermansia muciniphila $(p=0.037)$ and several Bacteroides members. Predictive functional analysis revealed overrepresentation of carbohydrate metabolism function in general $(p=0.0058)$ and glycosaminoglycan degradation $(p=0.015)$, fatty acid biosynthesis $(p=0.0082)$, and sugar (fructose, mannose) metabolism $(p=0.0065)$ in particular in the fecal microbiota of PM recipients compared to controls, and many of these functions were linked to Bacteroides spp. Pretreatment with PM not only diminished susceptibility to dextran sulfate sodium (DSS) to induced colitis severity (colon inflammation score; $p=0.029$ ), but also caused enhanced immune regulation through increasing Foxp3 + T-cells $(p=0.0108)$ and IL10- producing cells $(p=0.0159)$, and decreasing IFNy producing cells $(p=0.0224)$.

\section{Conclusions}

The use of PM as an oral dietary supplement effectively alters the composition of gut microbiota by enriching certain microbial communities such as Bacteroides spp. and carbohydrate metabolism function. These changes promote an anti-inflammatory/regulatory immune response which suppresses susceptibility to gut inflammation. Overall, this study demonstrates the prebiotic properties of PM and the potential benefits of its prolonged oral consumption to gut health.

\section{Introduction}

Prebiotic supplementation has long been used for promoting digestive health and regularity through manipulation of the gut microbiome [1, 2]. One group of such compounds are $\beta$-glucans (BGs), which are 
complex polymers of $D$-glucose held together primarily via $\beta-1,3$ glycosidic linkages, with differing patterns of bonds such as $\beta-1,4$ and $\beta-1,6$ linkages or branching distinguishing the different types of BGs $[3,4,5]$. These non-digestible molecules can be extracted from many sources, including yeast $[4,5,6,7]$, barley and oats $[8,9]$, and algae $[3,10]$. Many health benefits of these complex dietary polysaccharides (CDP), including lowering of low-density lipoprotein (LDL) cholesterol [11, 12], immune system stimulation and maintenance of immune homeostasis [3], attenuation and prevention of autoimmunity through increased anti-inflammatory cytokine production [4, 5], anti-tumor activity [13, 14], and shortchain fatty acid (SCFA) production [15] have been reported. Key immune system modulating health benefits observed with $\beta$-glucans, at least in part, appear to be through its direct binding to Dectin-1, a pathogen recognition receptor $[3,16]$. Our research on autoimmune diseases has additionally shown Dectin-1 or gut microbiota-dependent preventative and protective effects of systemic treatment with yeast $\beta$-glucan (YBG; $\beta-1,3 / 1,6-\mathrm{D}$-glucan) in type 1 diabetes (T1D) [17] and oral treatment with YBG in T1D [4] and colitis [5].

Previous reports, including ours, have found that YBG and barley $\beta$-glucan ( $\beta$-1,3/1,4-D-glucan) administration can significantly alter the gut microbiota in mouse models $[4,5]$ and humans [8], respectively. In mouse models of T1D and colitis, oral treatment with YBG enriched Bacteroidetes and Verrucomicrobia and diminished the phylum Firmicutes [4, 5]. At the genus level, Akkermansia spp. (Verrucomicrobia) and Parabacteroides spp. (Bacteroidetes) were found to increase significantly after treatment, while Oscillospira (Firmicutes) decreased after treatment. Functional predictions of the gut microbiota of yeast $\beta$-glucan-treated mice revealed an upregulation in functions linked to the utilization and metabolism of $\beta$-glucans after treatment. Treatment with other $\beta$-glucans have been found to stimulate and increase the levels of probiotics like bifidobacteria and lactobacilli in the gut, with oatderived $\beta$-glucan having a more profound influence compared to barley-derived $\beta$-glucan [18]. Similar shifts in Bacteroidetes to Firmicutes ratio were observed in human subjects who received high molecular weight $\beta$-glucan derived from barley [8]. Further, in silico predictions showed enhanced functions of microbiota related to glycan utilization and glycerolipid metabolism after treatment with these cereal derived $\beta$-glucans.

While yeast and cereal derived $\beta$-glucans have been studied in conjunction with their effects on the gut microbiota in relation to cardiovascular and immune health $[4,5,8,19]$, not much is known about the effects of a widely used microalgae-derived $\beta$-glucan, paramylon (PM; $\beta-1,3-D$-glucan) on the gut microbiota and gut immune function. PM is produced by Euglena gracilis as a storage molecule that exists primarily as crystallized granules within the cell [20,21,22]. One distinguishing feature of PM compared to other $\beta$-glucans is its high yield, typically making up at least $90 \%$ of the dry weight of the host cell [22]; hence, it is considered easier to extract a highly purified version of $\beta$-glucan. In a study examining obesity, inclusion of paramylon into a high fat diet improved obesity outcomes via increased relative levels of Bifidobacterium and Lactobacillus [23]. A study examining the effect of laminarin or paramylon [24] concluded that differences in digestive system and gut microbiome responses to different $\beta$-glucans are likely due to differences in size, weight, and solubility. 
In this study, we determined the progressive changes in microbiota structure and function in B6 mice during treatment with highly purified (>99\%) PM. We observed that prolonged oral treatment with PM significantly altered both the composition and function of the gut microbiota, with increases in Bacteroides and Akkermansia taxa and functions potentially linked to carbohydrate metabolism. Conversely, we found a decrease in the compositional levels and functions linked to members of Firmicutes after PM treatment. Importantly, pretreatment of mice with PM resulted in enhanced immune regulation and suppressed susceptibility to chemical induced colitis. These studies, for the first time, demonstrate the prebiotic properties of a microalgae derived $\beta$-1,3-D-glucan and how its consumption may influence the gut environment both microbiologically and immunologically.

\section{Materials And Methods}

\section{Mice and Treatments}

C57BL/6 (B6) mice were originally purchased from the Jackson Laboratory (Bar Harbor, ME) and bred within the specific pathogen-free (SPF) facility at the Medical University of South Carolina (MUSC). Mice were maintained on a standard autoclaved rodent diet and autoclaved water. MUSC's animal care and use committee approved the use of animals for this study. For microbiota analysis, female mice aged 8 weeks were administered either a $0.9 \%$ saline solution as control or $250 \mu \mathrm{g}$ of PM (Millipore-Sigma; cat\# 89862 ) in saline per day, for 45 consecutive days, via oral gavage. Fecal pellets were collected from individually housed mice at day 0 (prior to treatment), 15 days, 30 days, and 45 days post-treatment and stored at $-20^{\circ} \mathrm{C}$.

\section{Analysis of the impact of PM pretreatment on gut inflammation}

Additional cohorts of B6 mice were each administered either saline or $250 \mu \mathrm{g}$ PM suspension daily, via oral gavage, for 55 days. At day 45 , mice received $2.5 \%(\mathrm{w} / \mathrm{v}$ ) dextran sulfate sodium (DSS) to induce colitis until day 50 , when they were switched back to regular water for the remainder of treatment. Mouse body weight was measured on day 45 (pre-treatment with DSS) and every day thereafter. At day 55, mice were euthanized and their colons harvested and their lengths measured. Sections of the distal colon were subjected to hematoxylin and eosin (H\&E) staining and severity was evaluated as described previously [5]. Mesenteric lymph nodes (MLN) and colon tissues from control and PM treated mice were harvested for analysis of immune response to PM treatment. Fluorescence-activated cell sorting (FACS) analysis was performed on MLN cells that were stained for Foxp3 or MLN cells activated using PMA/lonomycin with Brefeldin A for $4 \mathrm{~h}$ and stained for different cytokines. From the colon tissues, immune cells were enriched from single cell suspensions as described before [4, 5] and cultured with anti-CD3 antibody for $24 \mathrm{~h}$ and then subjected to Luminex technology based multiplex assay to determine the concentration of cytokines. 


\section{S Ribosomal RNA Gene Sequencing and Bacterial Community Analysis}

DNA was extracted from fecal pellets as described previously $[4,5,25]$ and the V3-V4 region of the $16 \mathrm{~S}$ ribosomal RNA (rRNA) gene was sequenced on an Illumina MiSeq platform in the genomic center at MUSC. Taxonomic classifications and diversity analysis were performed on the resulting sequencing reads using the web-based sequencing analysis software MicrobiomeAnalyst with the Marker Data Profiling feature [26]. A BIOM file, metadata file, and phylogenetic tree file were uploaded and data filtering was performed. Normalization of the sequences by rarefying to the minimum library size $(5,164$ sequences) and total sum scaling was then performed to eliminate the disparity of unequal sequence depth. Taxonomic classification was performed against the GreenGenes database [27] and grouped into operational taxonomic units (OTUs) and classified based upon a $97 \%$ identity cutoff to references sequences within the database. Sequencing data was also used as described previously [5] for predictive functional analysis of Kyoto encyclopedia of genes and genomes (KEGG) orthologs using PICRUSt [28, 29], which assigned functions to three levels of functional categories, which were then analyzed using Statistical Analysis of Metagenomic Profiles (STAMP) software package [30]. Additional analysis of predicted functions was performed using the Global and Local Mapper features on the iVikodak platform [31].

\section{Statistical Analysis}

Statistical analyses including calculation and comparison of means, standard deviation (SD), and statistical significance ( $p$-value) were performed and figures constructed using EXCEL (Microsoft, Redmond, WA), STAMP [30], and GraphPad Prism (San Diego, CA) applications. Welch's $t$-tests were implemented in GraphPad Prism to test the significance between treatments unless otherwise states. $P$ values were corrected in STAMP using the Benjamini and Hochberg correction method [32]. The significance in a-diversity and $\beta$-diversity between samples was analyzed in MicrobiomeAnalyst via Mann-Whitney and permutational multivariate ANOVA (PERMANOVA) methods, respectively. Regarding immune system analyses, statistical significance was calculated via unpaired $t$-test for body at each time point, Fisher's exact test for comparing inflammation scores, and Mann-Whitney test for immune cell assays.

\section{Results}

Prolonged oral administration of PM alters the structure of gut microbiota

To determine the impact of consuming highly purified PM on gut microbiota, B6 mice were treated with this agent by oral gavage for 45 consecutive days. The pre- and post-treatment fecal samples from control and PM recipients were collected and examined for progressive changes in the profiles of microbial communities by $16 \mathrm{~S}$ rRNA gene sequencing. An analysis of alpha (a) and beta ( $\beta$ ) diversities revealed differences in the overall structure of gut microbiota of control and PM treated mice. Although 
gut microbiota appeared to be unaltered initially (15d), between day 15 and day 30 of treatment, the average richness of the gut microbiota significantly decreased in both control $(p<0.001)$ and PM treated mice $(p<0.001)$, based on Chao1 richness estimator (Fig. 1A). While this change appears to be, in part, due to age dependent maturation and/or unknown changes in the environment, the degree of decrease in richness was significantly greater in PM treated mice compared to controls at both $30(p=0.0302)$ and 45 days $(p=0.0064)$. Further, Shannon index estimates (Fig. 1B) revealed a significant decrease in diversity at day 30 in both control $(p=0.0101)$ and PM treated mice $(p<0.0001)$. In PM treated mice, diversity decreased significantly further at day $45(p=0.034)$ from day 30 . Diversity was significantly lower in PM treated mice compared to controls at both day $30(p=0.045)$ and $45(p=0.018)$.

$\beta$ diversity was measured to evaluate the overall differences in community structure between control and PM treatment groups via Principal Coordinate Analysis (PCOA) using Bray-Curtis index with PERMANOVA. These analyses revealed spatially distant clustering of most of the day 30 and day 45 samples from control and PM treated groups compared to pre-treatment and 15 day time-points (Fig. 1C). In PM treated mice, compared to controls, samples from day 45 showed more spatial separation from day 30 samples. When samples grouped according to the duration of treatment were compared, significantly distinct clustering was observed between control and PM treated mice at both day $30(p=0.018)$ and day $45(p=$ 0.013) time-points (Fig. 1D). These observations suggest that while the gut microbiota in B6 mice used in this study appeared to have undergone maturation, PM treatment had a significant impact on its overall structure and composition.

PM treatment results in changes in the fecal microbial community profile

Next, we examined if 16S rRNA gene sequencing data of control and PM treated mice show differences in the profiles of gut microbial communities. Compilation of OTUs to different taxonomical levels revealed distinct patterns in gut microbial composition between control and PM treatments. At the phylum level (Fig. 2A \& B), the gut microbiota in general was primarily comprised of Bacteroidetes (31.6 to $64.7 \%$ ), Firmicutes (1.5 to $26.7 \%$ ), Proteobacteria (8.1 to $16.2 \%$ ), and Verrucomicrobia (0.4 to $45.1 \%$ ). While Verrucomicrobia was the only phylum that showed progressive increase in abundance between day 0 and day 45 ( $p=0.0062$ for control and $p=0.0005$ for PM treated), the increase on day 45 was significantly higher in PM treated mice compared to controls $(p=0.002)$. Compared to day 0 , other key changes observed at day 45 in PM treated mice include significant decreases in Bacteroidetes $(p=0.0004)$, Firmicutes $(p=0.0059)$, and Proteobacteria $(p=0.0179)$. No significant differences in these phyla were observed between days 0 and 45 in control mice. Importantly, the day 45 abundance of Firmicutes was significantly lower in PM treated mice compared to their control counterparts $(p=0.0147)$.

At the genus level (Fig. 2C \& D), the most abundant taxa represented in the gut included Akkermansia, Bacteroides, Blautia, and Parabacteroides. Changes in fecal microbiota over time include significant increases in Akkermansia ( $p=0.0056$ for control and $p=0.0005$ for PM treated mice), Luteolibacter ( $p=$ 0.005 for control and $p=0.002$ for PM treated mice), and Rubritalea ( $p=0.009$ for control and $p=0.00095$ for PM treated mice), and significant decreases in Clostridium ( $p=0.0158$ for control and $p=0.0186$ for 
PM treated mice), Flavobacterium ( $p=0.006$ for control and $p=0.008$ for PM treated mice), and Oscillospira ( $p=0.026$ for control and $p=0.006$ for PM treated mice) between days 0 and 45 . Importantly, significant time-dependent changes observed only in PM treated mice include decreases in Blautia spp. $(p=0.0018)$ and Parapedobacter $(p=0.0087)$. Significant differences also existed at day 45 between control and PM treated mice at the genus level (Fig. 2D). These included significantly higher levels of Akkermansia $(p=0.0024)$ and Bacteroides $(p=0.0106)$ and lower levels of Parabacteroides $(p=0.02)$ and Blautia $(p=0.02)$ in PM recipient mice compared to controls.

Prolonged treatment with PM causes selective enrichment of microbial communities

Data presented in Figs. 1 \& 2 suggested, in addition to some age dependent changes, progressive changes in the gut microbiota due to PM treatment. Since progressive changes were noticeable with samples collected on day 30 and later, additional comparisons of microbial community profiles of control and PM treated groups at these time points were made. As observed in heat maps of Fig. 3A, relatively higher abundance of Verrucomicrobia and lower abundances of a major phylum Firmicutes and minor phyla were observed at days 30 and 45 , but statistically significant differences were detected only with day 45 samples. At genus level, similar trends in the increase of Bacteroides, Akkermansia, Luteolibacter, and Rubritalea and decrease of Blautia, Oscillospira, and Parabacteroides were observed in PM recipient mice (Fig. 3B).

Further compilation of OTUs at species level revealed a greater number of significant time dependent changes in the microbial communities of PM treated mice compared to controls (Supplemental Fig. 1). Further, differences in the abundances of higher numbers of communities over time (day 30 vs day 45) were observed within PM treated mice when directly compared to controls (Fig. 3C \& D). Notably, Akkermansia muciniphila showed significant increase within the gut microbiota in mice of both groups, with a more pronounced increased in PM treated mice. Other communities that increased in the PM treated mice at day 30 and day 45, compared to pre-treatment time-point or control group at day 45 include an unclassified Bacteroides sp., a Prevotella sp., and Bacteroides acidifaciens. In PM treated mice, there were significant decreases of greater than $5 \%$ in an unclassified member of the Clostridiales order at day $45(p=0.035)$ and an unclassified member of the family S24-7 of phylum Bacteroidetes at days $30(p=0.011)$ and $45(p=0.0081)$. Overall, these analyses show progressive changes/enrichment of microbial communities during PM treatment.

PM treatment causes altered function of gut microbiota

Predicted functional profiles of fecal microbiota were generated from the OTU table using PICRUSt for control and PM treated groups and analyzed for statistically significant differences. Regarding specific functions, no significant differences were observed in the functional levels in control mice between day 0 and 30 (data not shown). At both day 30 and 45, gut microbiota of the PM group showed a greater number of significantly different functions compared to control mice (Supplemental Fig. 2). Comparisonbetween the different functions of microbiota revealed significant overrepresentation of carbohydrate metabolism at both day 30 and 45 in PM treated mice compared to controls (Fig. 4A \& B). 
Overall, gut microbiota of PM treated mice showed significantly higher level of functions related to sugar processing and metabolism at both day 30 and 45, including fructose and mannose metabolism, glycosaminoglycan degradation, and pentose processing and utilization. As anticipated, microbiota of PM treated mice at day 45 showed more altered functions compared to their day 30 counterparts. These included functions related to various carbohydrate processing and metabolism, fatty acid biosynthesis and metabolism, as well as certain amino acid metabolisms. Functions significantly downregulated at day 30 and/or day 45 include aminoacyl-tRNA biosynthesis, C5-branched dibasic acid metabolism, translation proteins, and methane metabolism. Representative functions that were significantly upregulated in PM treated mice were analyzed further using the Local Mapper tool on iVikodak to determine which taxa were contributing the most to these particular functions. As shown in Supplemental Figs. 3, 4, \& 5, functions such as fructose and mannose metabolism, glycosaminoglycan degradation, and fatty acid biosynthesis, which are upregulated in PM treated mice, are linked primarily to the Bacteroides genus. In PM recipient mice, there is a reduction in each of the examined functions linked to Firmicutes taxa compared to controls. Overall, these analyses, along with the microbial community profile data, show an association between PM treatment and progressive changes in the structure and function of gut microbiota.

Pretreatment with PM diminishes susceptibility to DSS induced colitis

Our recent report showed that YBG, a structurally distinct $\beta$-glucan, can suppress colitis susceptibility when the mice were pre-treated [5]. To determine if PM treatment and associated structural and functional changes in gut microbiota impact colitis susceptibility, B6 mice were given PM for 55 consecutive days and DSS during the days 45-50, as shown in Fig. 5A, and monitored for colitis associated loss of body weight. In addition, colon length and colonic inflammation were determined after euthanasia on day 55. As observed in Fig. 5B, PM treated mice were relatively less susceptible to weight loss compared to control mice. Significant difference in the body weight between control and PM treated groups was observed starting day 8 post-DSS treatment initiation. Although colon lengths of control and PM treated mice, upon euthanasia, were comparable (Fig. 5C), overall histological injury scores in DSStreated mice that received PM, compared to control group, were significantly lower $(p=0.029)$ (Fig. 5D). Overall, these observations show that pre-treatment with PM for considerable duration can diminish susceptibility to colitis.

PM treated and control mice with colitis showed distinct immune characteristics

Since PM fed mice showed less severe colitis compared to controls, immune cells from their gut associated tissues were examined for the phenotypic properties to realize if PM treatment related events caused immune modulation. As observed in Fig. 6A, Foxp3 $+T$ cell frequencies were profoundly higher in the MLN of PM fed mice compared to control mice $(p=0.018)$. Importantly, PMA/ionomycin stimulation ex vivo, followed by FACS analyses, showed that IL $10+T$ cell frequencies were significantly higher $(p=$ $0.0226)$ and IFNY + T cell frequencies were lower $(p=0.0449)$ in PM fed mice. These observations were validated by multiplex cytokine assay that showed similar differences in the levels of IL10 and IFNY 
secreted by colonic immune cells from PM treated and control mice $(p=0.0119$ and $p=0.0224$ respectively), in response to ex vivo activation using anti-CD3 antibody (Fig. 6B). Moreover, albeit not statistically significant, IL17 response was also relatively lower in PM treated mice compared to controls. These results suggest that long-term PM treatment modulates the immune cell phenotype in gut mucosa.

\section{Discussion}

Previous work has shown $\beta$-glucans to be important modulators of the host immune response $[3,4,5,16$, $17,33]$. While our studies have shown profound effects of YBG on both the host's gut microbiota as well as autoimmune disease/colitis outcomes [4,5], impact of another widely used $\beta$-glucan dietary supplement, PM of microalgae origin, on gut microbiota and immune function is largely unknown. The current study shows a significant prebiotic effect of PM upon prolonged oral administration on both shaping the gut microbiota and reducing susceptibility to gut inflammation. These changes include the altering of abundance of key bacterial taxa, influencing the community structure of gut microbiota, increasing the levels of microbial functions related to carbohydrate metabolism, and improving disease outcomes with colitis through reduced inflammation and increased production of Tregs and antiinflammatory cytokines.

Prolonged oral administration of PM caused progressive changes in the structure of gut microbiota. At the phylum level, PM treated mice had significantly higher levels of Verrucomicrobia and significantly lower levels of Firmicutes. Previous studies using YBG have similarly found Verrucomicrobia to significantly increase and Firmicutes levels to profoundly decrease [4, 5]. In contrast to YBG treatment, however, we did not observe significant changes in Bacteroidetes upon PM treatment. Differences in $\beta$ glucan structure may contribute to this observed difference, as the differing physical structure and properties of $\beta$-glucan can potentially alter the number of gut microbiota that preferentially utilize them [24]. While the Bacteroides genus showed profound increase upon PM treatment, the lack of noticeable effect on Bacteroidetes in PM treated mice seems to be primarily due to significantly less abundance in the S24-7 family, a common member in the mammalian gut, capable of metabolizing a diverse array of polysaccharides that seems to illicit a response by the innate immune system [34]. It appears that S24-7 family members do not have the ability to degrade PM and use it as an energy source for their growth and the reduction in their abundance may also be contributing to diminished susceptibility of PM recipient mice to gut inflammation.

The significant increase in Verrucomicrobia, particularly Akkermansia muciniphila, upon treatment with different types of $\beta$-glucans indicates an enrichment of bacteria in the mucosal lining of the intestines [35], which suggests either a prominent role of these bacteria in the metabolism of these complex carbohydrates or that $\beta$-glucan treatment contributes to increased mucus production in the gut that enriches this organism. Importantly, treatment with PM significantly increased the abundance of Bacteroides compared to controls. Several known species, including $B$. acidifaciens, $B$. ovatus, and $B$. uniformis, as well as unclassified Bacteroides spp. were found to be significantly more prevalent in PM treated mice. Bacteroides spp. in the gut are typically associated with the production of SCFA like 
butyrate and acetate, which have important roles in the digestive and immune system function [36]. Therefore, decreased susceptibility to colitis in PM treated mice may be linked to the increased levels of these bacteria. PM treatment also appears to downregulate the levels of certain bacteria within the gut, including Oscillospira spp., similar to YBG $[4,5]$. This genus has been reported to be a prominent member of the gut microbiome and appears to be linked with dietary habits and lower body fat in humans [37]. These bacteria are suggested to be unable to effectively metabolize complex fiber and tend to decrease upon higher supplementation of a plant-based diet [37], which could explain the observed decrease in their levels upon treatment.

Although, the richness and diversity (a diversity) of the gut microbiota decreased over time in both control and PM treated mice, this decrease in PM treated mice was significantly more pronounced than in control mice. This suggests that degradation of this CDP enriches certain bacteria significantly, reducing the diversity within the mouse gut. Similar to YBG treatment [5], PM treatment induced a decrease in richness of at least 150 estimated species within the gut microbiota. $\beta$ diversity analysis showed significantly different community structures in control and PM treated mice at day 30 and 45 , indicating that $\beta$-glucan treatment causes a shift in the community composition and structure within the gut microbiota. Tighter clustering observed in PM treated mice is indicative of the prebiotic effects of this CDP, which selectively enrich for potentially beneficial bacteria which contributes to diminished susceptibility to gut inflammation.

In agreement with the structural changes in the gut microbiota, PM treatment showed a significant effect on its predictive functions, evidenced by significant overrepresentation of functions related to carbohydrate metabolism, fatty acid biosynthesis, and glycan biosynthesis and metabolism. This is consistent with utilization of CDPs and subsequent SCFA production $[15,18,38]$. Most of the overrepresented functions observed in the gut microbiota of control mice were linked to Firmicutes, primarily to the genus Proteiniborus. Conversely, most of the overrepresented metabolic pathways observed in the gut microbiota of PM treated mice were linked to Bacteroides, which has many species capable of SCFA synthesis and modulation of the immune system [5, 36, 39].

Importantly, PM treatment-associated shaping of the composition and function of the gut microbiota appears to impact the immune system leading to suppression of susceptibility to DSS -induced colitis. Our data showing a higher retention of body weight and a markedly lower inflammation in the colon of mice pretreated with PM, in addition to the significant increase in Treg frequencies and the antiinflammatory cytokine IL10 indicate that PM treatment associated changes in gut microbiota structure and function are immune regulatory and host beneficial.

\section{Conclusions}

Oral administration of PM as a dietary supplement is effective in altering the gut microbiota in composition, community structure, and function and in diminishing colitis susceptibility. The evidence presented herein suggests the prebiotic nature of PM oral treatment, which significantly alters the 
function of the gut microbiota toward enhanced carbohydrate metabolism and contributes to reduced susceptibility to gut inflammation upon DSS-induced colitis. The decreased level of IFNY and increased anti-inflammatory response upon PM treatment lend support toward this notion. Nevertheless, further studies using gnotobiotic and microbiota depleted mice are needed in the future to fully understand the importance of specific microbial communities in diminishing susceptibility to gut inflammation upon dietary treatment using PM.

\section{Abbreviations}

BG

B-glucan

CDP

complex dietary polysaccharides

DSS

dextran sulfate sodium

FACS

Fluorescence-activated cell sorting

H\&E

hematoxylin and eosin

KEGG

Kyoto encyclopedia of genes and genomes

LDL

low-density lipoprotein

MLN

Mesenteric lymph nodes

OUT

operational taxonomic units

PICRUSt

Phylogenetic Investigation of Communities by Reconstruction of Unobserved States

PM

paramylon

PMA

phorbol myristate acetate

SCFA

short-chain fatty acid

SPF

specific pathogen-free

STAMP

Statistical Analysis of Metagenomic Profiles

T1D 
type 1 diabetes

YBG

yeast $\beta$-glucan

\section{Declarations}

\section{ETHICS APPROVAL AND CONSENT TO PARTICIPATE}

Not applicable.

\section{CONSENT FOR PUBLICATION}

Not applicable.

\section{AVAILABILITY OF DATA AND MATERIALS}

"Please contact author for data requests."

\section{COMPETING INTERESTS}

The authors declare that they have no competing interests.

\section{FUNDING}

This work was supported by internal funds from MUSC, National Institutes of Health $(\mathrm{NIH})$ grants: R21Al133798 (NIAID), and R21Al133798 and R21Al136339-02S1 Administrate supplements (Office of Dietary Supplements) to CV. CV is the guarantor of this work and as such, has full access to all the data in the study and takes responsibility for integrity of the data and accuracy of data analysis.

\section{AUTHORS' CONTRIBUTIONS}

H.T. analyzed data and wrote manuscript, R.G. and R.B. researched the data; C.V. designed experiments, researched and analyzed data, and reviewed/edited manuscript.

\section{ACKNOWLEDGEMENTS}

The authors are thankful to the Histology core of Pathology department of MUSC for the histology service, and Genomic center of MUSC for 16S rRNA gene sequencing service.

\section{AUTHORS' INFORMATION}

None.

\section{References}


1. Gibson RG, Roberfroid MB. Dietary modulation of the human colonic microbiota: introducing the concept of prebiotics. J Nutr. 1995;125:1401-1412.

2. Schley PD, Field CJ. The immune-enhancing effects of dietary fibres and prebiotics. Br J Nutr. 2002;87, Suppl 2:S221-S230.

3. Nakashima A, Yamada K, Iwata O, Sugimoto R, Atsuji K, Ogawa T, Ishibashi-Ohgo N, Suzuki K. $\beta$ glucan in foods and its physiological functions. J Nutr Sci Vitaminol. 2018;64:8-17.

4. Gudi R, Perez N, Johnson BM, Sofi MH, Brown R, Quan S, Karumuthil-Melethil S, Vasu C. Complex dietary polysaccharide modulates gut immune function and microbiota, and promotes protection from autoimmune diabetes. Immunology. 2019;157:70-85.

5. Gudi R, Suber J, Brown R, Johnson BM, Vasu C. Pretreatment with Yeast-derived complex dietary polysaccharides suppresses gut inflammation, alters the microbiota composition, and increases immune regulatory short-chain fatty acid production in C57BL/6 mice. J Nutr. 2019;nxz328.

6. Kodama N, Komuta K, Nanba H. Effect of maitake (Grifola frondosa) D-fraction on the activation of NK cells in cancer patients. J Med Food. 2003;6:371-377.

7. Manners DJ, Masson AJ, Patterson JC. The structure of a $\beta$-(1à3)-d-glucan from yeast cell walls. Biochem J. 1973;135:19-30.

8. Wang Y, Ames NP, Tun HM, Tosh SM, Jones PJ, Khafipour E. High molecular weight barley ß-glucan alters gut microbiota toward reduced cardiovascular disease risk. Front Microbiol. 2016;7:129.

9. Johansson L, Virkki L, Mauna S, Lehto M, Ekholm P, Varo P. Structural characterization of water soluble $\beta$-glucan of oat bran. Carbohydr Polym. 2000;42:143-148.

10. Monfils AK, Triemer RE, Bellairs EF. Characterization of paramylon morphological diversity in photosynthetic euglenoids (Euglenales, Euglenophyta). Phycologia. 2011;50:156-169.

11. Wolever TMS, Tosh SM, Gibbs AL, Brand-Miller J, Duncan AM, Hart V, Lamarche B, Thomson BA, Duss R, Wood PJ. Physicochemical properties of oat $\beta$-glucan influence its ability to reduce serum LDL cholesterol in humans: a randomized clinical trial. Am J Clin Nutr. 2010;92:723-32.

12. Whitehead A, Beck EJ, Tosh S, Wolever TM. Cholesterol-lowering effects of oat beta-glucan: a metaanalysis of randomized controlled trials. Am J Clin Nutr. 2014;100:1413-1421.

13. Ohno N, Furukawa M, Miura NN, Adachi Y, Motoi M, Yadomae T. Antitumor $\beta$-glucan from the cultured fruit body of Agaricus blazei. Biol Pharm Bull. 2001;24:820-828.

14. Watanabe T, Shimada R, Matsuyama A, Yuasa M, Sawamura H, Yoshida E, Suzuki K. Antitumor activity of the $\beta$-glucan paramylon from Euglena against preneoplastic colonic aberrant crypt foci in mice. Food Funct. 2013;4:1685-90.

15. Miyamoto J, Watanabe K, Taira S, Kasubuchi M, Li Xuan, Irie J, Itoh H, Kimura I. Barley $\beta$-glucan improves metabolic condition via short-chain fatty acids produced by gut microbial fermentation in high fat diet fed mice. PLoS ONE. 2018;13:e0196579.

16. Plato A, Willment JA, Brown GD. C-type lectin-like receptors of the dectin-1 cluster: ligands and signaling pathways. Int Rev Immunol. 2013;32:134-156. 
17. Karumuthil-Melethil S, Gudi R, Johnson BM, Perez N, Vasu C. Fungal $\beta$-glucan, a Dectin-1 ligand, promotes protection from type 1 diabetes by inducing regulatory innate immune response. $\mathrm{J}$ Immunol 2014;193:3308-3321.

18. Jayachandran M, Chen J, Chung SSM, Xu B. A critical review on the impacts of $\beta$-glucans on gut microbiota and human health. J Nutr Biochem. 2018;61:101-110.

19. Ryan PM, London LEE, Bjorndahl TC, Mandal R, Murphy K, Fitzgerald GF, Shanahan F, Ross RP, Wishart Ds, Caplice NM, Stanton C. Microbiome and metabolome modifying effects of several cardiovascular disease interventions in apo- $\mathrm{E}^{-/-}$Microbiome. 2017;5:30.

20. Marchessault RH, Deslandes Y. Fine structure of (1à3)- $\beta$-D-glucans: curdlan and paramylon. Carbohydr Res. 1979;75:231-242.

21. Chuah CT, Sarko A, Deslandes Y, Marchessault RH. Triple-helical crystalline structure of curdlan and paramylon hydrates. Macromolecules. 1983;16:1375-1382.

22. Barsanti L, Vismara R, Passarelli V, Gualtieri P. Paramylon ( $\beta$-1,3-glucan) content in wild type and WZSL mutant of Euglena gracilis. Effects of growth conditions. J Appl Phycol. 2001;13:59-65.

23. Okouchi R, E S, Yamamoto K, Ota T, Seki K, Imai M, Ota R, Asayama Y, Nakashima A, Suzuki K, Tsuduki T. Simultaneous intake of Euglena gracilis and vegetables exerts synergistic anti-obesity and ant-inflammatory effects by modulating the gut microbiota in diet-induced obese mice. Nutrients. 2019;11:204.

24. Kuda T, Enomoto T, Yano T. Effects of two storage $\beta$-1,3-glucans, laminarin from Eicenia bicyclis and paramylon from Euglena gracili, on cecal environment and plasma lipid levels in rats. J Funct Foods. 2009;1:399-404.

25. Johnson BM, Gaudreau MC, Al-GI-Gadban MM, Gudi R, Vasu C. Impact of dietary deviation on disease progression and gut microbiome composition in lupus-prone SNF1 mice. Clin Exp Immunol. 2015;181:323-37.

26. Dhariwal A, Chong J, Habib S, King IL, Agellon LB, Xia J. MicrobiomeAnalyst: a web-based tool for comprehensive statistical, visual and meta-analysis of microbiome data. Nucleic Acids Res. 2017;45:W180-W188.

27. DeSantis TZ, Hugenholtz P, Larsen N, Rojas M, Brodie EL, Keller K, Huber T, Dalevi D, Hu P, Andersen GL. Greengenes, a Chimera-checked 16S rRNA gene database and workbench compatible with ARB. Appl Environ Microbiol. 2006;72:5069-5072.

28. Wixon J, Kell D. The Kyoto encyclopedia of genes and genomes - KEGG. Yeast. 2000;17:48-55.

29. Langille MGI, Zaneveld J, Caporaso JG, McDonald D, Knights D., a Reyes J, Clemente JC, Burkepile DE, Vega Thurber RL, Knight R, Beiko RG, Huttenhower C. Predictive functional profiling of microbial communities using 16S rRNA marker gene sequences. Nature Biotechnol. 2013;8:1-10.

30. Parks DH, Tyson GW, Hugenholtz P, Beiko RG. STAMP: statistical analysis of taxonomic and functional profiles. Bioinformatics. 2014;30:3121-3124. 
31. Nagpal S, Haque MM, Singh R, Mande SS. iVikodak - a platform and standard workflow for inferring, analyzing, comparing, and visualizing the functional potential of microbial communities. Front Microbiol. 2019;9:3336.

32. Benjamini $Y$, Hochberg $Y$. Controlling the false discovery rate: a practical and powerful approach to multiple testing. J R Stat Soc B. 1995;57:289-300.

33. Suzuki I, Hashimoto K, Ohno N, Tanaka H, Yadomae T. Immunomodulation by orally administered $\beta$ glucan in mice. Int J Immunopharmacol. 1989;11:761-769.

34. Ormerod KL, Wood DLA, Lachner N, Gellatly SL, Daly JN, Parsons JD, Dal'Molin CGO, Palfreyman RW, Nielsen LK, Cooper MA, Morrison M, Hansbro PM, Hugenholtz P. Genomic characterization of the uncultured Bacteroidales family S24-7inhabiting the guts of homeothermic animals. Microbiome. 2016;4:36.

35. van Passel MWJ, Kant R, Zoetendal EG, Plugge CM, Derrien M, Malfatti SA, Chain PSG, Woyke T, Palva A, de Vos WM, Smidt $\mathrm{H}$. The genome of Akkermansia muciniphila, a dedicated intestinal mucin degrader, and its use in exploring intestinal metagenomes. PLOS ONE. 2011;6:e16876.

36. Chen T, Long W, Zhang C, Liu S, Zhao L, Hamaker BR. Fiber-utilizing capacity varies in Prevotellaversus Bacteroides-dominated gut microbiota. Sci Rep. 2017;7:2594.

37. Konikoff T, Gophna U. Oscillospira: a central, enigmatic component of the human gut microbiota. Trends Microbiol. 2016;24:523-524.

38. Lam KL, Cheung PCK. Non-digestible long chain beta-glucans as novel prebiotics. Bioact Carbohydr Dietary Fibre. 2013;2:45-64.

39. Gorvitovskaia A, Holmes SP, Huse SM. Interpreting Prevotella and Bacteroides as biomarkers of diet and lifestyle. Microbiome. 2016;4:15.

\section{Figures}


A

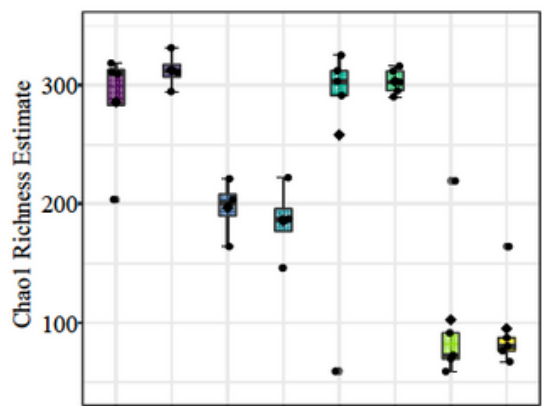

Day \begin{tabular}{llllllll}
0 & 15 & 30 & 45 & 0 & 15 & 30 & 45 \\
\hline
\end{tabular}

B
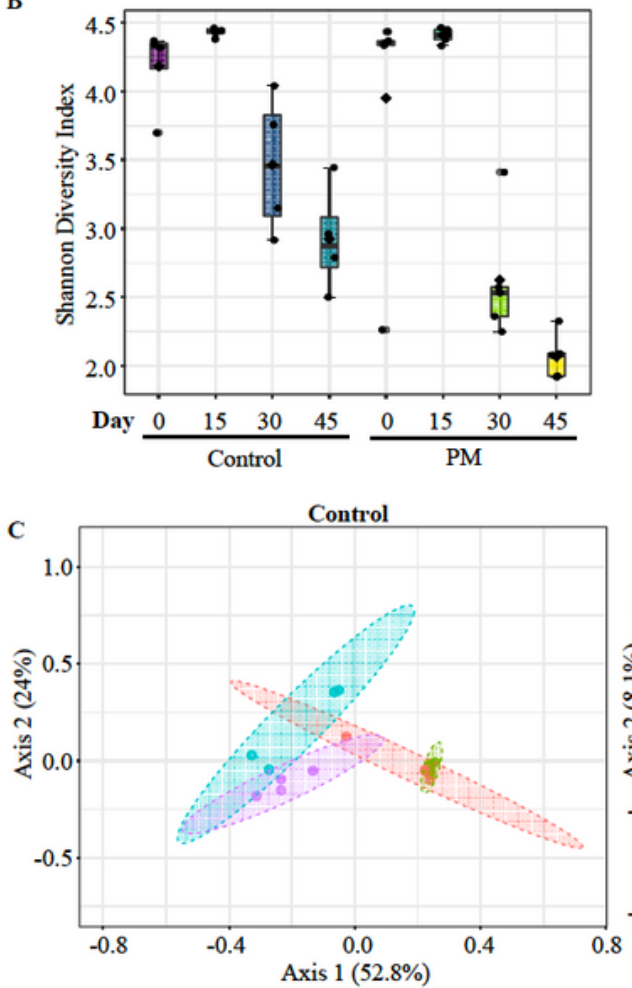

D

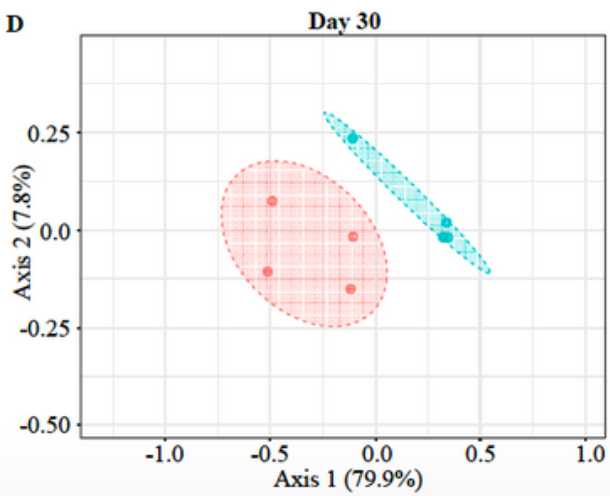

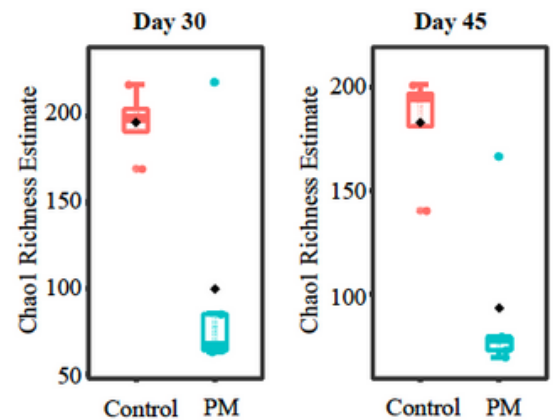
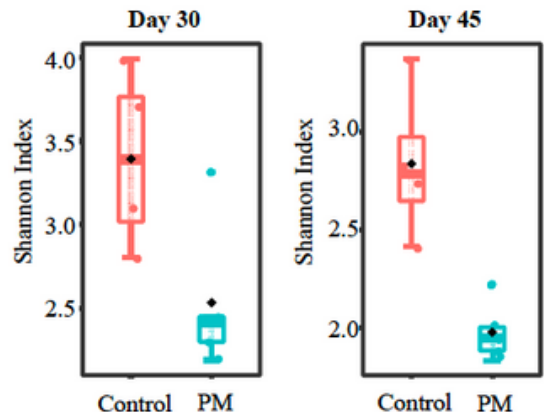

PM

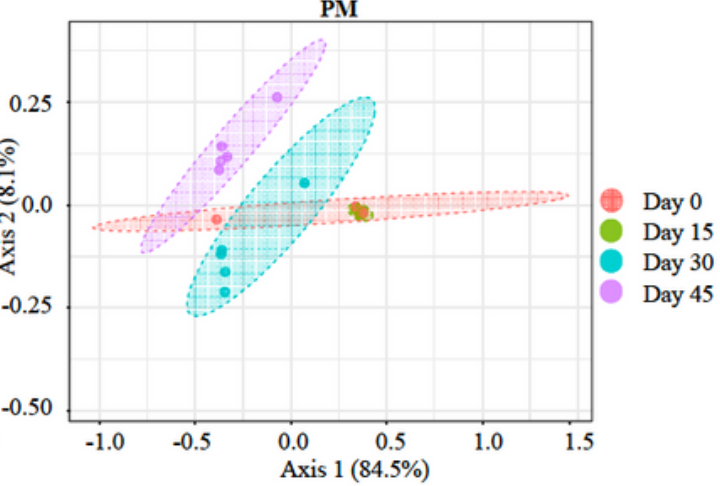

Day 45

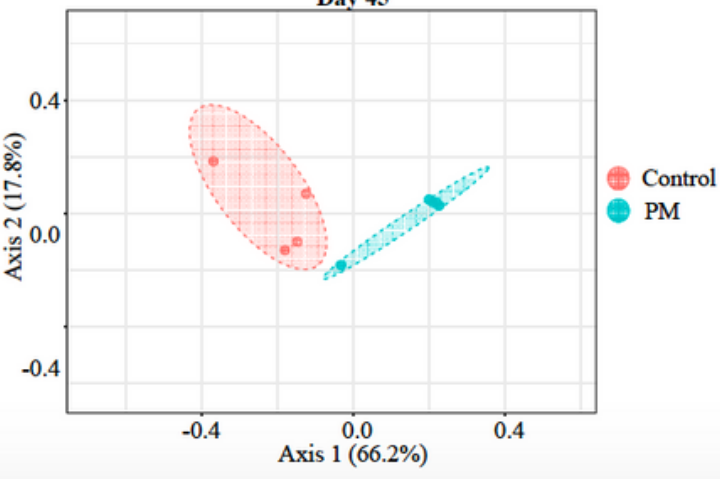

\section{Figure 1}

$a$ and $\beta$ diversity of the fecal microbiota in control and PM-treated mice. 16S rRNA gene sequencing data of fecal samples from control $(n=4)$ and PM treated $(n=5)$ mice before (day 0$)$ and various time-points (days 15,30 and 45 ) during the treatment were subjected to $\nabla$ and $\otimes$ diversity analyses. Chao 1 estimates (A) and Shannon indices (B) were employed to evaluate richness and diversity within each sample, respectively. Statistical significance was assessed using t-tests with FDR correction. Principal coordinate 
analysis (PCoA) was used to analyze the community structure differences between samples over time for each treatment (C) and between treatments at day 30 and day 45 (D) using Bray-Curtis dissimilarity and statistical significance was evaluated using PERMANOVA.

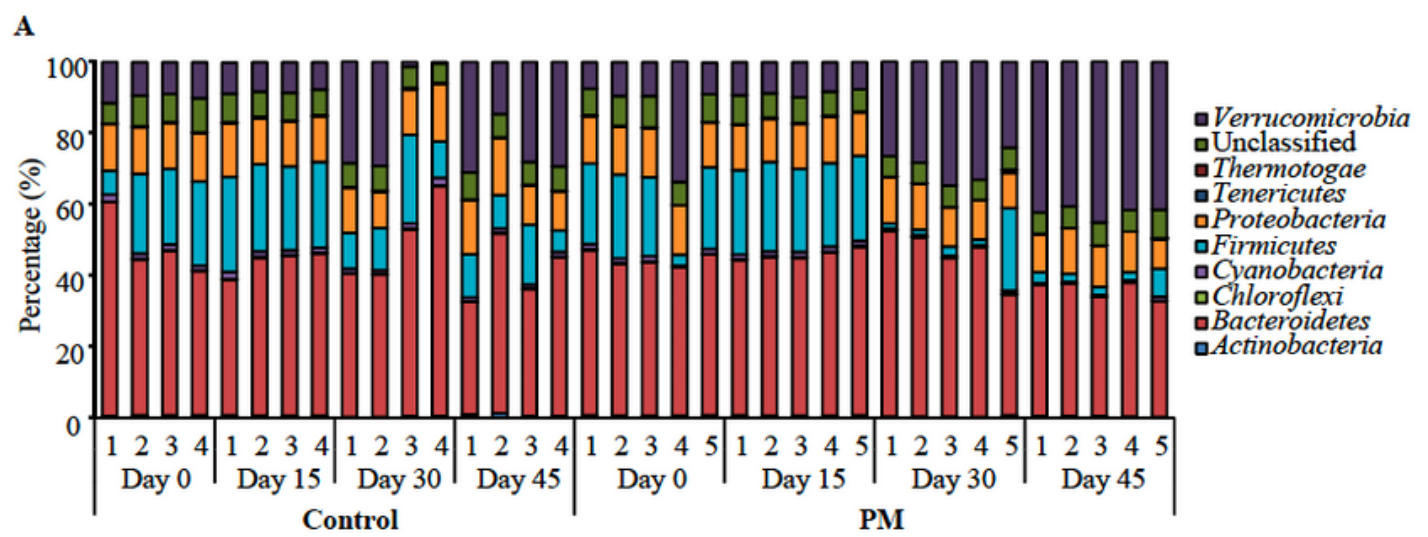

B
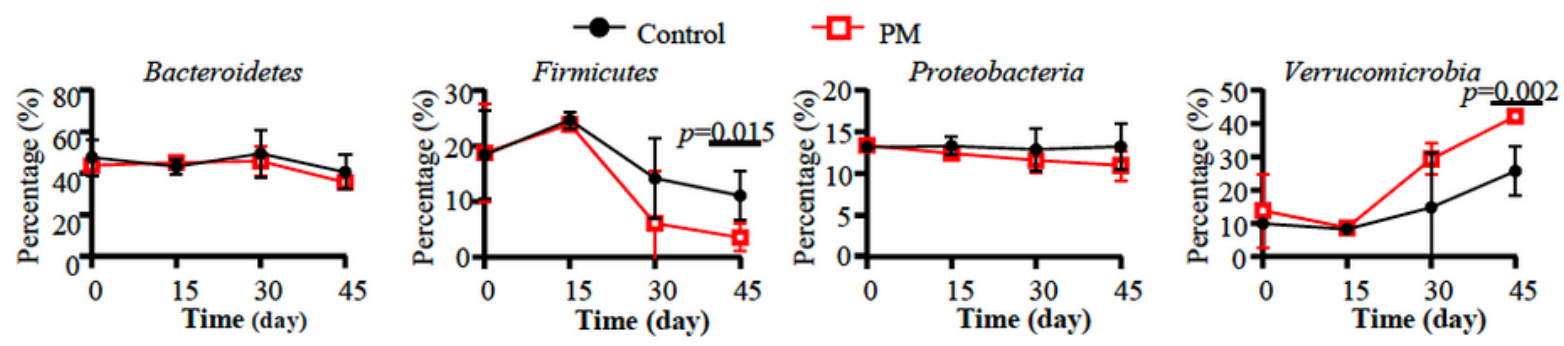

C

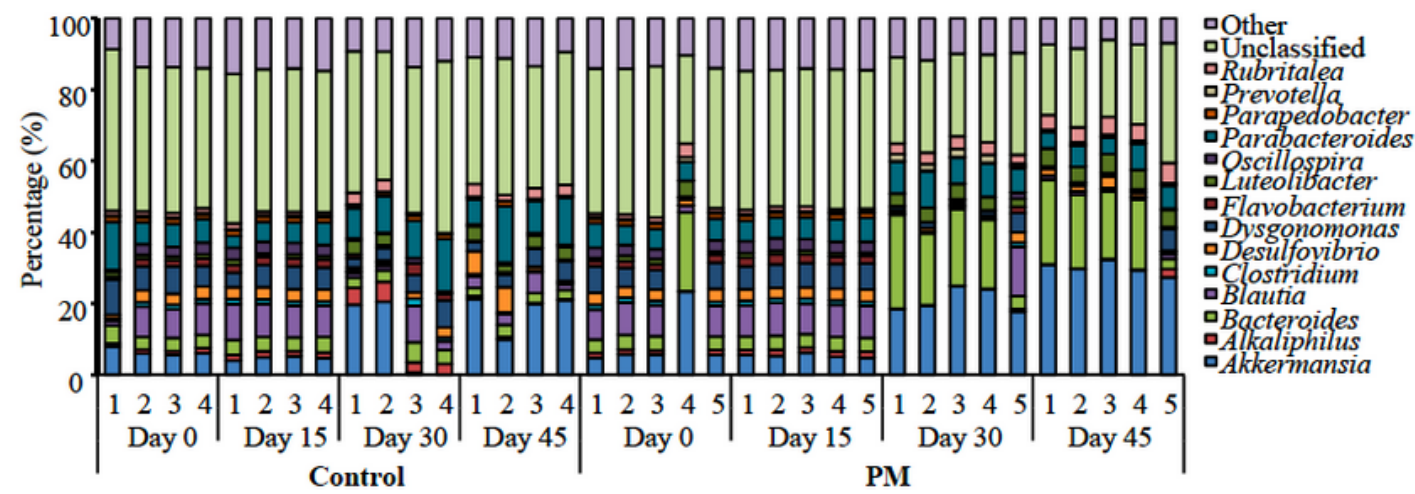

D
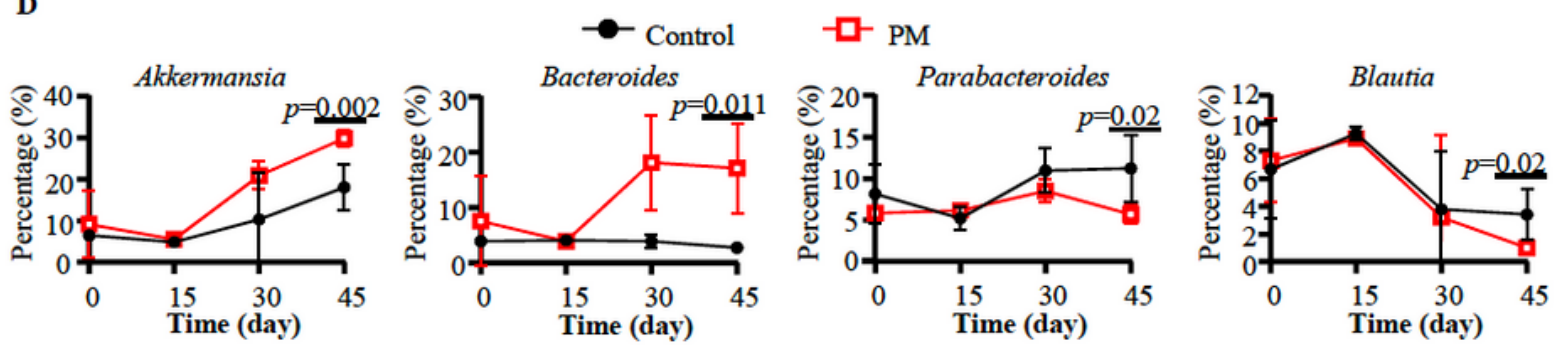

Figure 2

Time-dependent changes of fecal microbiota in control and PM treated mice. Relative abundances of microbial communities at the Phylum and Genus levels were determined in fecal samples collected from 
control $(n=4)$ and PM treated $(n=5)$ mice before (day 0$)$ and various time points (days 15,30 , and 45 ) during the treatment. Analysis of time-dependent changes at phylum level (A) and in major phyla (B), and at genus level (C) and in major genera (D) between control and PM treated mice are shown. p-values were determined using unpaired t-test on GraphPad Prism.

A
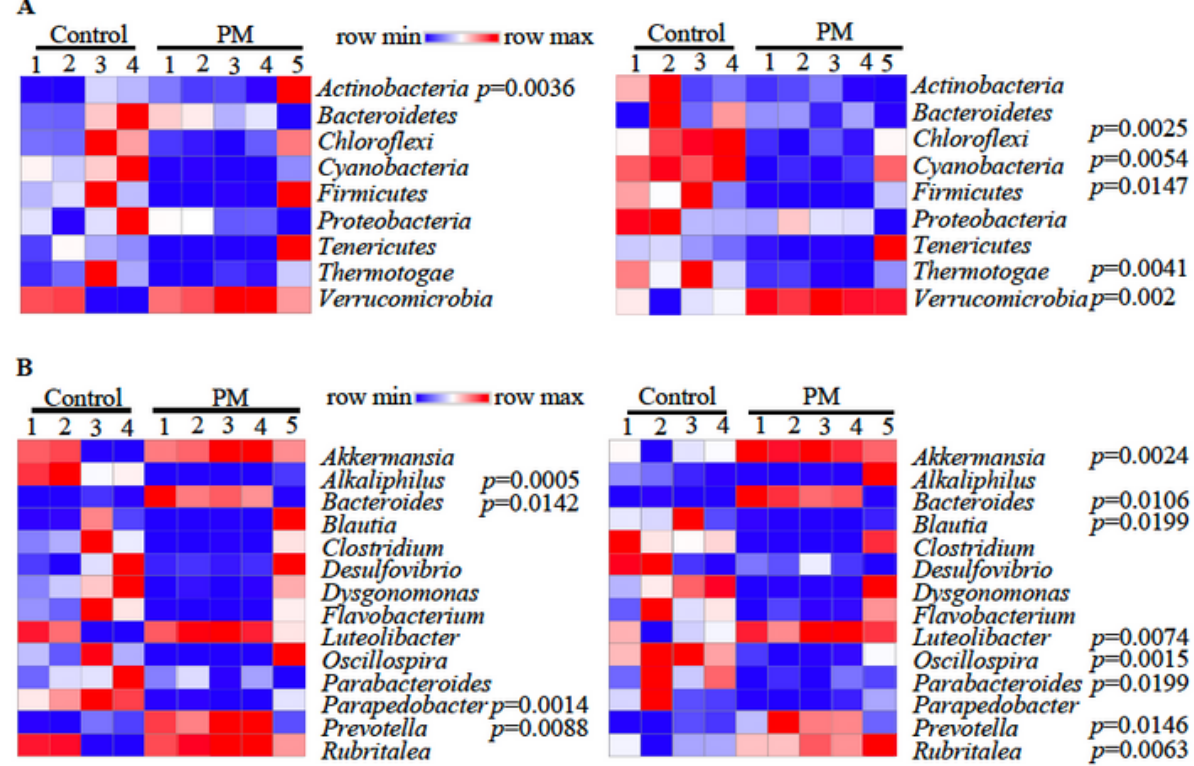

row $\min =$ row $\max$
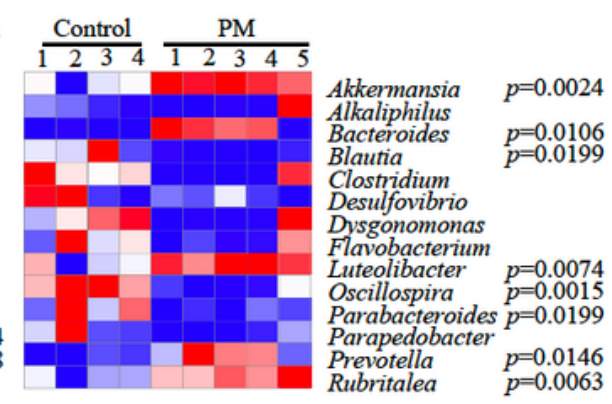

Akkermansia

Alkaliphilus $\quad p=0.0005$

Bacteroides $\quad p=0.0142$

Blautia

Clostridium

Dysgonomonas

Flavobacterium

Luteolibacter

Parabacteroides

Parapedobacter $p=0.001$

Prevotella $\quad p=0.0088$

Rubritalea

Rubritalea

$p=0.0063$
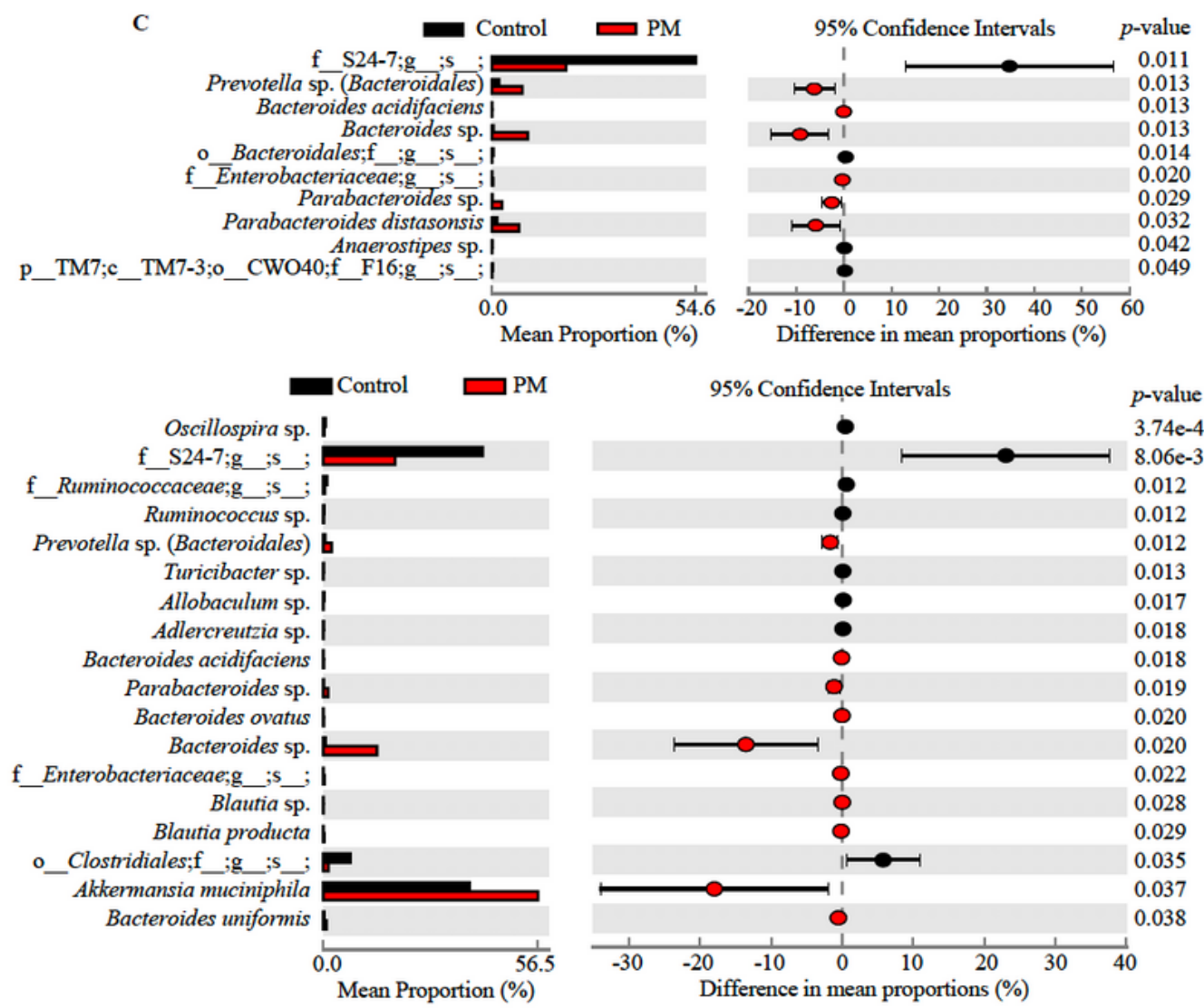

Figure 3 
Comparison of fecal microbiota in control and PM treated mice. 16S rRNA gene sequencing data described in figures $1 \& 2$ were analyzed for relative abundances of microbial communities at the phylum $(A)$ and genus (B) levels in fecal samples collected from control and PM treated mice on day 30 (left panels) and day 45 (right panels). Heat maps were generated using Morpheus

(https://software.broadinstitute.org/morpheus), p-values by unpaired t-test. Relative abundances of microbial communities at species level (C) were also determined for fecal samples collected from control and PM treated mice on day 30 (upper panel) and day 45 (lower panel) employing STAMP. FDR corrected p-values (Welch's t-test) are shown. 
Control $\square \mathrm{PM}$

Carbohydrate Metabolism

0.0

RNA polymerase

Fructose \& Mannose Metabolism Pentose \& Glucuronate Interconversions

Other Ion-coupled Transporters Translation Proteins

Aminoacyl-tRNA Biosynthesis Thiamine Metabolism Unclassified Carbohydrate Metabolism
Ribosome Biogenesis C5-branched Dibasic Acid Metabolism
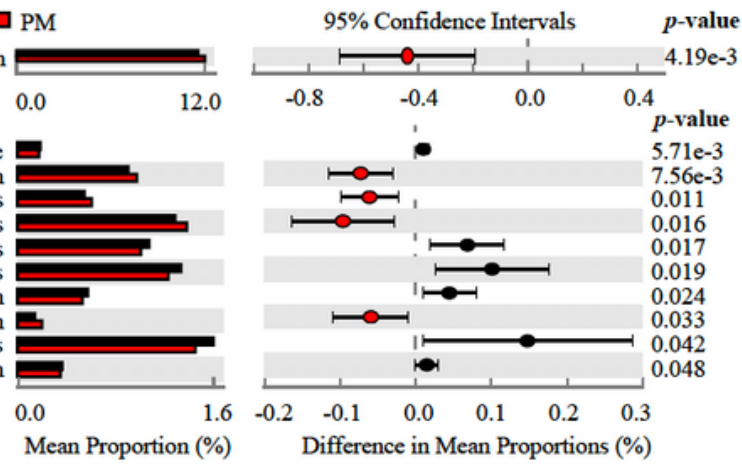

Control $\square \mathrm{PM}$

Cellular Processes \& Signaling Carbohydrate Metabolism Metabolism Enzyme Families Lipid Metabolism Xenobiotics Biodegradation \& Metabolism Nucleotide Metabolism

Genetic Information Processing
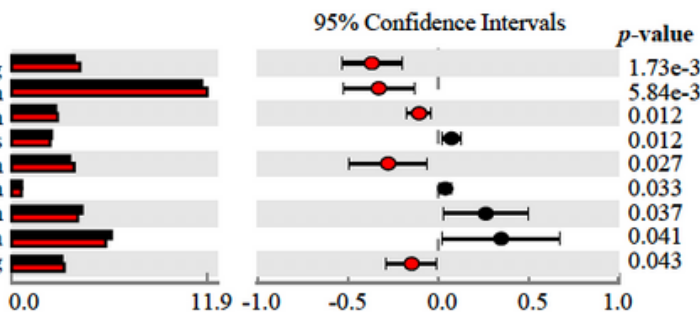

Valine, Leucine, Isoleucine Degradation Pores Ion Channels Propanoate Metabolism Fructose \& Mannose Metabolism Glutathione Metabolism Fatty Acid Biosynthesis Lipid Biosynthesis Proteins Phenylalanine Metabolism enylalanine Metabolism Aminoacyl-tRNA Biosynthesis Transcription Machinery Glycosaminoglycan Degradation Glyoxylate \& Dicarboxylate Metabolism Tyrosine Metabolism Sulfur Metabolism Base Excision Repair Pyruvate Metabolism Sulfur Relay System Other Ion-coupled Transporters Unclassified Carbohydrate Metabolism Lysine Degradation Methane Metabolism Metabolism of Cofactors and Vitamins Inorganic Ion Transport \& Metabolism Lysine Biosynthesis Pyrimidine Metabolism Glycerophospholipid Metabolism Lipopolysaccharide Biosynthesis Proteins Ubiquinone \& Other Terpenoid-quinone Biosynthesis
Glycosyltransferases

C5-branched Dibasic Acid Metabolism

Lipopolysaccharide Biosynthesis

Glycolysis, Gluconeogenesis

Selenocompound Metabolism

Bacterial Secretion System Peptidases Riboflavin Metabolism Protein Folding \& Processing N-Glycan Biosynthesis Replication, Recombination, \& Repair Proteins
Other Metabolism Homologous Recombination RNA transport Chromosome Pentose Phosphate Pathway
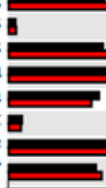

$\begin{array}{lllllll}0.0 & 2.1 & -0.4 & -0.2 & 0.0 & 0.2 & 0.4\end{array}$

p-value

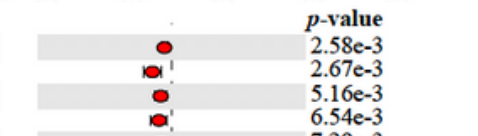

$\begin{array}{ll}0 & 7.29 \mathrm{e}-3 \\ a_{1} & 8.22 \mathrm{e}-3 \\ 0.26 \mathrm{e}-3\end{array}$

$9.26 \mathrm{e}-3$

0.012
0.013

0.014

0.015

0.015

0.017

0.017
0.019

0.020

0.022

0.022

0.023

0.023

0.023
0.024

0.024
0.025

0.026

0.026

0.027

0.029
0.030

0.030

0.030
0.031

0.031
0.032

0.032

0.032

0.033

0.033

0.034

0.034

0.035
0.035

0.035
0.038

0.038
0.038

0.038

0.040

0.044

0.048

Mean Proportion (\%) Difference in Mean Proportions (\%)

Figure 4

Predictive functional analysis of fecal microbiota in control and PM treated mice. Predictive functional analysis of OTU data described in figure $1 \& 2$ was done using PICRUSt and analyzed and visualized in STAMP showing designated functions at the $2 \mathrm{nd}(\mathrm{A})$ and $3 \mathrm{rd}(\mathrm{B})$ level of functional hierarchy at day 30 (upper panels) and 45 (lower panels) between control and PM treated mice. FDR corrected p-values (Welch's t-test) are shown. 

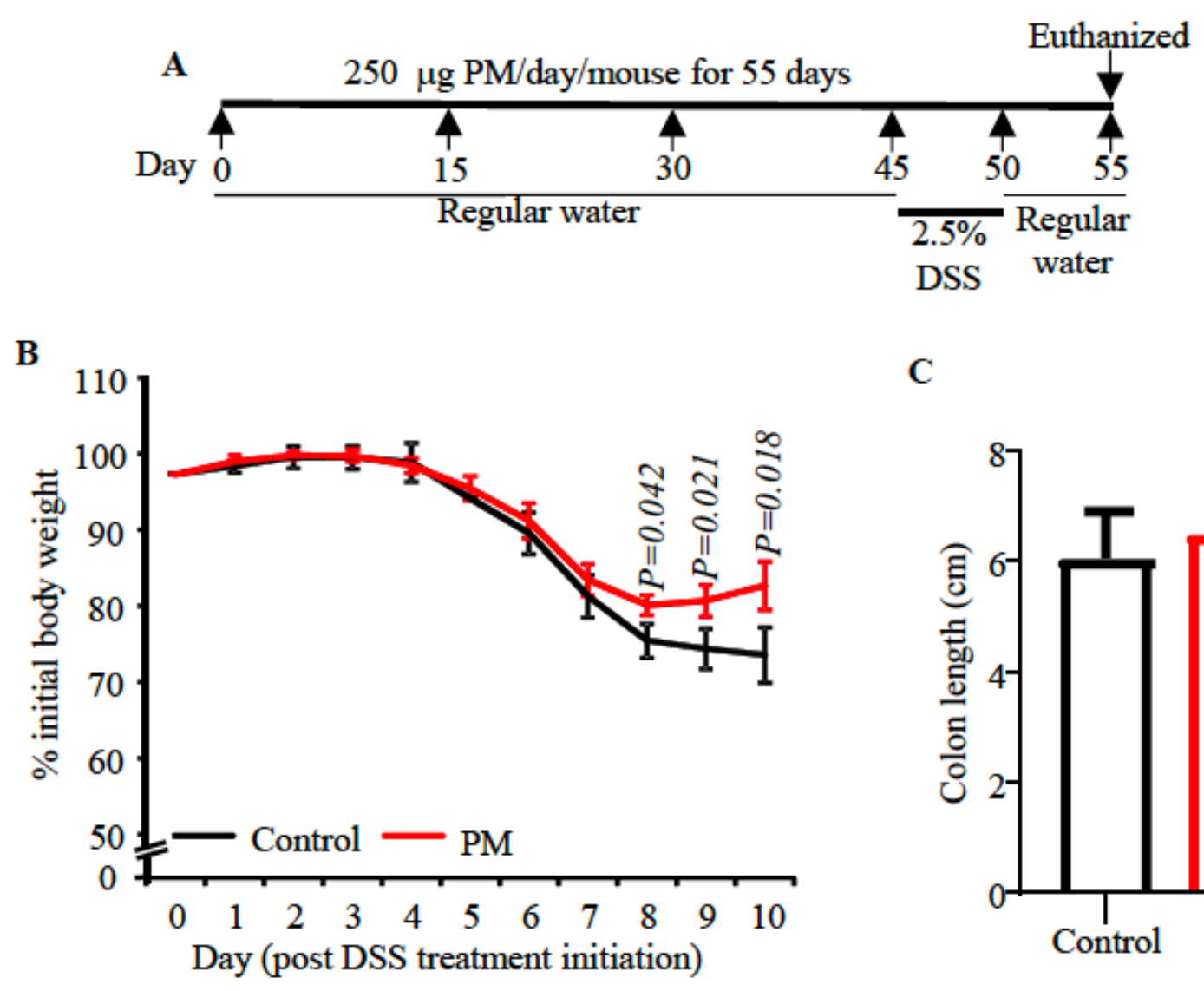

C

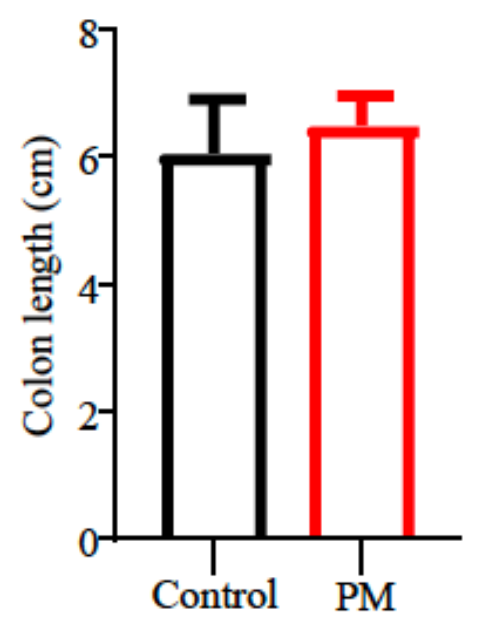

D
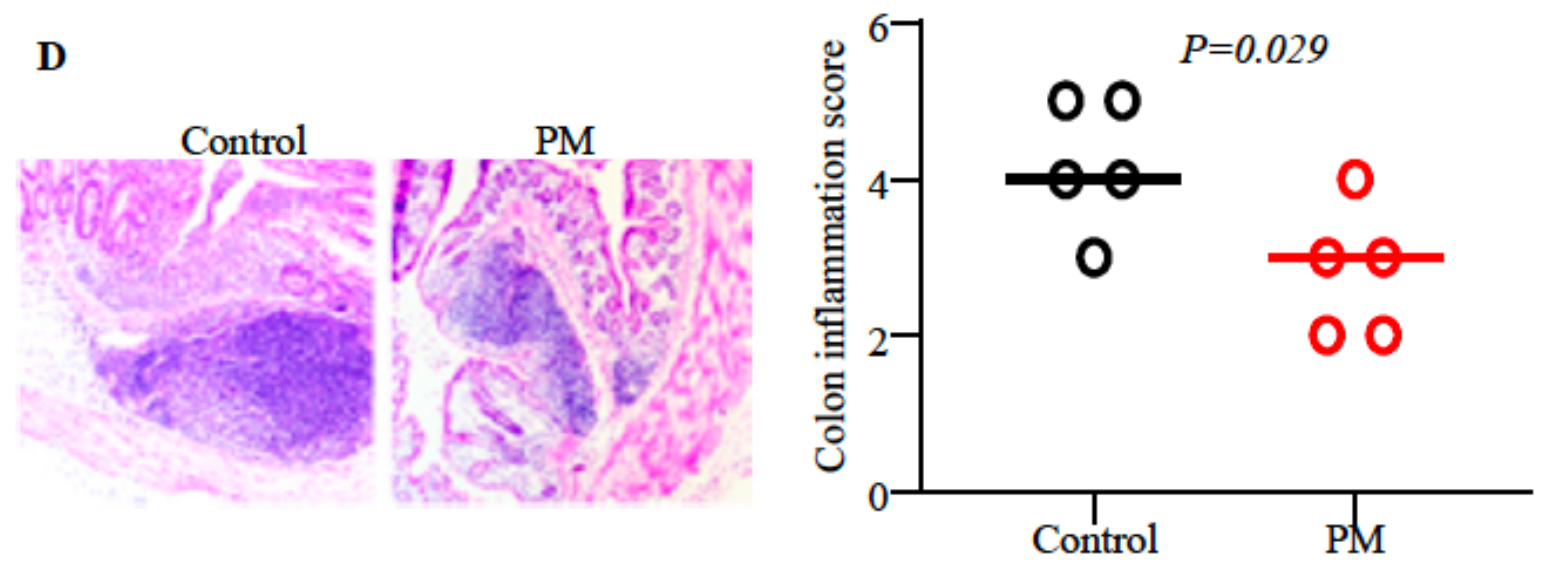

Figure 5

Impact of pre-treatment with PM on susceptibility to DSS-induced colitis. B6 mice were treated as depicted in panel A. Body weight of individual mice was measured starting pre-DSS treatment (day 45) every day and mean $\pm S D$ values of changes in total body weights, relative to initial body weight $(n=5 /$ group) are shown (B). $p$-value by unpaired t-test for each time-points. Mice were euthanized on day 55 , colons were harvested, and their length was measured and mean $\pm S D$ values of length of colons from 
5 mice/group are shown (C). H\&E stained distal colon sections were evaluated for the degree of inflammation and representative images of sections (left panel) and mean $\pm S D$ values of inflammation severity scores of 5 mice/group (right panel) are shown (D). p-values by Fishers' exact test comparing number of mice with grade $\leq 3$ and $\geq 4$ inflammation scores.

A
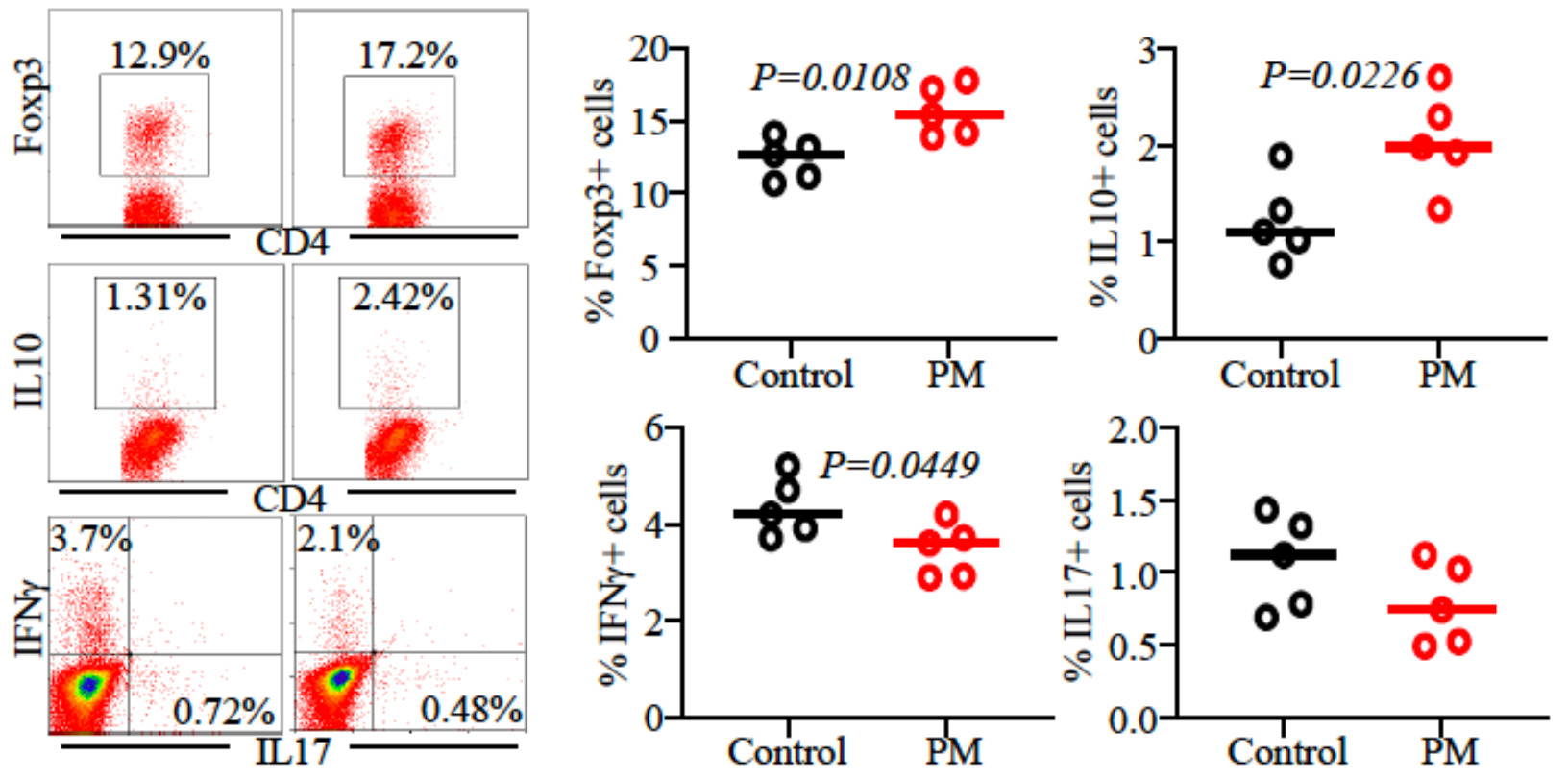

\section{B}
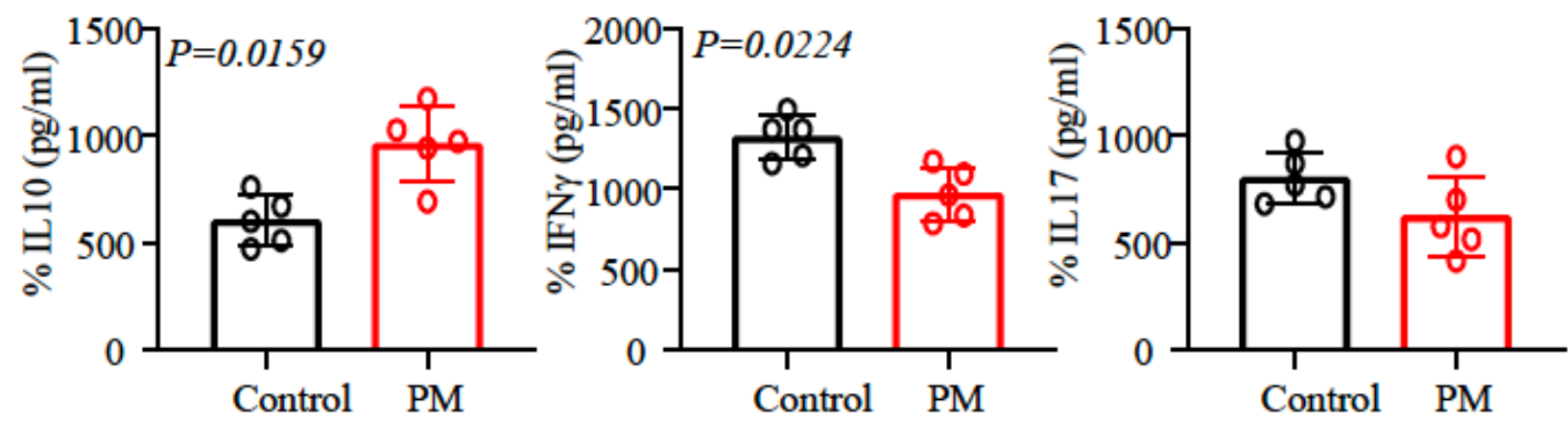

Figure 6

Impact of pre-treatment with PM on immune cells. B6 mice were treated as depicted in Fig. 5A. A) MLN cells were stained for Foxp3 and subjected to FACS analysis. Cells were also activated using $\mathrm{PMA} /$ lonomycin in the presence of Brefeldin $\mathrm{A}$ for $4 \mathrm{~h}$ and subjected to intracellular staining for cytokines and analyzed by FACS. CD4+ population was gated for determining cell frequencies. Representative FACS plots (left panel) and mean $\pm S D$ frequencies of cells positive for indicated specific factors, 5 mice/group 
tested individually in duplicate, (right panel) are shown. B) Immune cells were enriched from single cell suspension of colon tissue and cultured in the presence of anti-CD3 antibody for $24 \mathrm{~h}$ and the spent media were subjected to Luminex technology based multiplex assay. Mean $\pm S D$ values of concentrations of indicated cytokines produced by cells from 5 mice/group tested individually are shown. p-values by Mann-Whitney test.

\section{Supplementary Files}

This is a list of supplementary files associated with this preprint. Click to download.

- SupplementalFigures.pdf 